\title{
Bonding strength of 3D printed silicone and titanium retention magnets for maxillofacial prosthetics application
}

\author{
Sebastian Spintzyk ${ }^{a}$, Sophia Brinkmeier ${ }^{a}$, Fabian Huettig ${ }^{b}$, Alexey Unkovskiy ${ }^{c},{ }{ }^{*}$ \\ a Section Medical Materials Science and Technology, Tuebingen University Hospital, Tuebingen, Germany \\ ${ }^{b}$ Department of Prosthodontics at the Centre of Dentistry, Oral Medicine, and Maxillofacial Surgery with Dental School, Tuebingen University Hospital, Tue- \\ bingen, Germany \\ ' Department of Prosthodontics, Geriartric Dentistry and Craniomandibular Disorders, Charité - Universitätsmedizin Berlin, Corporate Member of Freie Univer- \\ sität Berlin, Humboldt - Universität zu Berlin, Berlin, Germany \\ d Department of Dental Surgery, Sechenov First Moscow State Medical University, Moscow, Russia
}

\begin{abstract}
Purpose: To assess the bonding between conventional and additively manufactured silicone elastomers and cylindrical retention titanium magnets for anchorage of facial prostheses.

Methods: The customized titanium retention magnets were embedded in conventional and additively produced silicone blocks without primer application $(n=20)$ and with two commercially available primers $G 611(n=20)$ and A304 $(n=20)$ applied onto the magnet surface. The pull out test was performed in the universal testing machine using $45^{\circ}$ and $90^{\circ}$ angulation and the pull out strength was measured for each group. Additionally the SEM images of the pulled out magnets' surface were obtained and the amount of residual silicone onto the magnet surface was quantified.

Results: Significantly higher pull out strength values $(p<0.05)$ were revealed for $90^{\circ}$ specimens $\left(0.11-0.17 \pm 0.01 \mathrm{~N} / \mathrm{mm}^{2}\right)$ compared to the $45^{\circ}$ group $\left(0.03 \pm 0.02 \mathrm{~N} / \mathrm{mm}^{2}\right)$. The pull out test with primer revealed no significant differences between the $\mathrm{G} 611$ and A 304 primers in the additive group. However, significantly $(p<0,05)$ higher values were observed for conventional specimens in the A304 group $\left(1.10 \pm 0.21 \mathrm{~N} / \mathrm{mm}^{2}\right)$ compared to the G611 group $\left(0.59 \pm 0.27 \mathrm{~N} / \mathrm{mm}^{2}\right)$.

Conclusion: The application of both used primers may be an acceptable technical option for the anchorage of retention titanium magnets in silicone facial prostheses, produced additively in a fully digital workflow.
\end{abstract}

Keywords: Facial prostheses; Nasal prostheses; Auricular prostheses; Silicone prostheses; Additive manufacturing

Received 15 January 2021, Accepted 31 May 2021, Available online 18 September 2021

\section{Introduction}

Prosthetic rehabilitation of facial disfigurements plays a big role in terms of patients' functional and social reestablishment and returns them to the accustomed way of life[1]. For decades silicone facial prostheses have been used to provide the wound seal and restore the former anatomy[2]. Until recent times such silicone prostheses have been manufactured in conventional workflow. Since computeraided design (CAD) and computer-aided manufacturing (CAM) have been evolving medicine, this has touched also upon the field of maxillofacial rehabilitation[3,4]. Thus, various technical approaches for facial prostheses manufacturing have been introduced, employing digital data capture, virtual prosthesis design and its subsequent materialization using additive manufacturing (AM)[5-7]. The latest techniques imply the direct printing of definitive silicone and siliconelike prostheses based on their virtual construction directly, avoiding

DOI: https://doi.org/10.2186/jpr.JPR_D_21_00019

*Corresponding author: Alexey Unkovskiy, Department of Prosthodontics, Geriartric Dentistry and Craniomandibular Disorders, Charité - Universitätsmedizin Berlin, Corporate Member of Freie Universität Berlin, Humboldt - Universität zu Berlin, Aßmannshauser Str. 4-6, 14197 Berlin, Germany.

E-mail address: alexey.unkovskiy@charite.de

Copyright: @ 2021 Japan Prosthodontic Society. All rights reserved. any additional steps, such as mold making and prototyping[8,9]. This approach has been initially used for the production of a provisional prosthesis[10]. Recently, the production of a definitive silicone prosthesis was shown in a fully digital workflow, utilizing the drop-ondemand technique[11].

A "fully digital workflow" of facial prostheses implies also the virtual planning, design and integration of retention elements[12,13]. Conventionally, the commercial titanium (cpTi) magnets are anchored in the prosthesis bulk mechanically with the help of retention rings. A later integration of such magnets into completely printed prosthesis bulk is not possible without damaging its structure. Alternatively, the retention can be provided with acrylic resin substructure, which is housing the cpTi and is integrated into prosthesis bulk (Fig. 1A). The adhesion between various resin types and maxillofacial elastomers has been thoroughly investigated, and in general such substructures provide sufficient surface for an efficient bonding[14-16]. However, a virtual design of such substructure in a fully digital workflow is time consuming and requires additional printing hardware for its fabrication[11]. Furthermore, in some clinical cases, where the space is limited, integration of additional retention elements may be restricted. For this reason, anchoring of cpTi adhesively within the prosthesis bulk would be a valid technical approach (Fig 1B). 


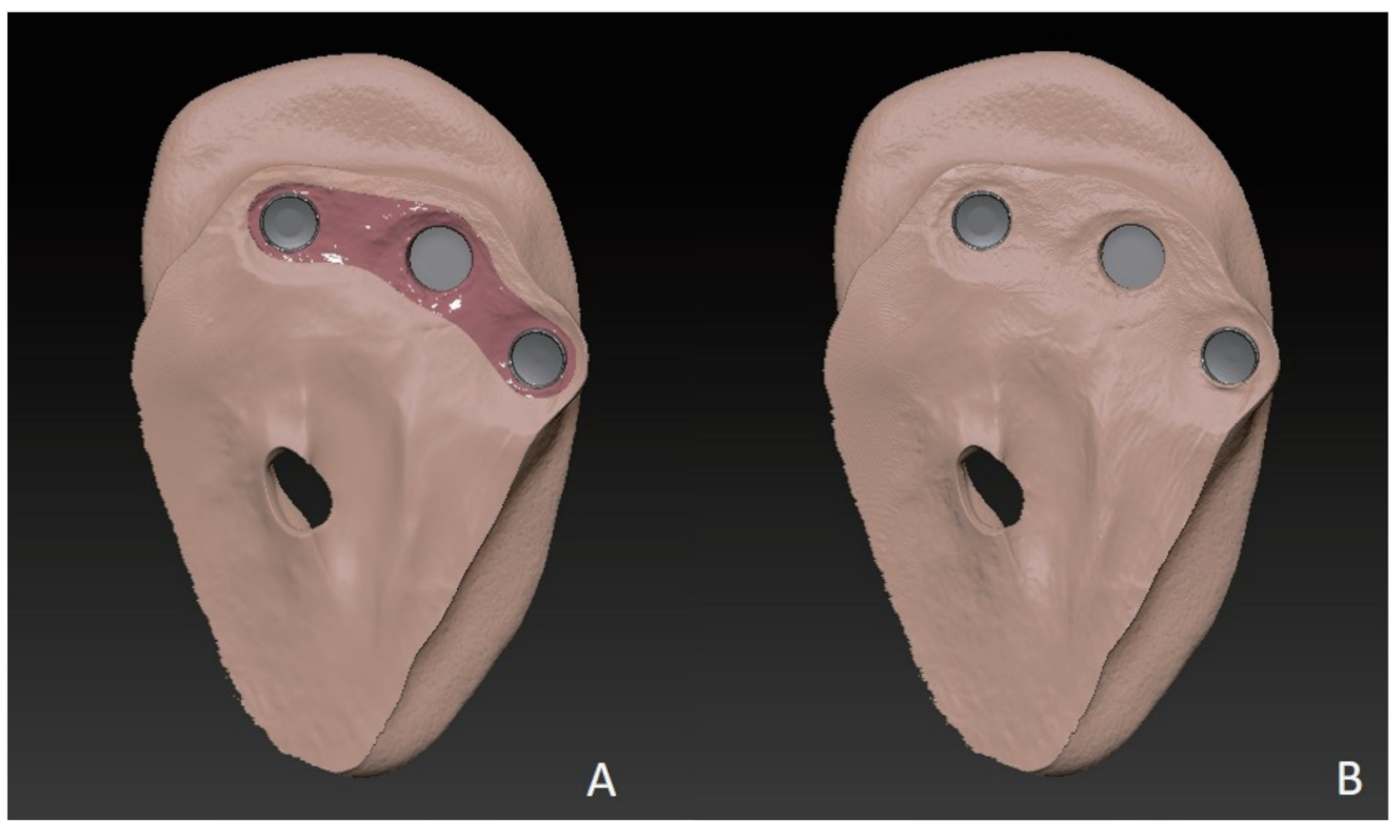

Fig. 1. Two magnet retention concepts within the silicone prosthesis bulk; A: by means of an acrylic superstructure adhesively bonded into the silicone; B: retention elements directly bonded into the silicone.

However, in the topical literature only two studies were found by the authors dealing with the bond strength between conventional silicone materials and cpTi addressing the application of various primers, too[17,18]. Since the rapid manufacturing of silicone prostheses may become a valid treatment approach, it necessitates a new protocol for anchoring of retention elements. For this reason it appears reasonable to investigate the ability of adhesively anchored cpTi's in the 3D printed silicone bulk to withstand a pull out force during insertion and removal of prosthesis.

Thus, the aim of the present study was to assess the bonding capacity of additively manufactured silicone by quantifying the pull out strength between cpTi and the directly printed silicone prosthesis bulk in comparison to conventional injection molded medical grade silicone. Additionally, the effect of two commercially available silicone-metal primers on the magnets retention was also investigated. The null hypothesis of this study was that there would be no differences in pull out strength between conventionally and additively manufactured silicones to cpTi without and with application of two various primers.

\section{Materials and Methods}

\subsection{Specimen design and fabrication}

2.1.1. Test specimen design

The customary retention magnets (X-line, Steco GmbH, Hamburg, Germany) with extended body to $10 \mathrm{~mm}$ were chosen as attachment surrogate in this study. A retention element was designed with a transfixion for the pull out test (Fig. 2A). The finished design of the test magnet was milled by the manufacturer (Steco GmbH, Hamburg, Germany) from the same material as the customary X-line magnets are made of (Fig. 2B). A total of 60 specimens were fabricated. The cylindrical area of the magnet design was sandblasted as the customary available $\mathrm{X}$-line magnet.

\subsubsection{Conventional group}

For production of silicone blocks a cylindrical hollow retention form of $20 \mathrm{~mm}$ in diameter and $6.5 \mathrm{~mm}$ in height with a cover plate were designed in CAD software (Fusion 360, Autodesk, San Rafael, USA) and printed with the stereolithography (SLA) (Form 2, Formlabs, Somerville, CA) method from grey resin material (Grey V4, Formlabs, Somerville, CA) (Fig. 3A). A medical-grade conventional silicone (Bredent, Senden, Germany, LOT 450417) was poured into the printed form and the cover plate was put on top of it. The specimen was submerged into the cover transition and fixed with a retention bar, which ensured the same specimen depth and position (Fig 3B). Thereafter this assembly was put into an oven (Universaloven UN, Memmert, Schwabach, Germany) in order to polymerize the silicone following the manufacturers specification $\left(60^{\circ} \mathrm{C}, 2\right.$ hours). The group "conventional-without-primer" encompassed 20 specimen (Fig 3C).

\subsubsection{Additive group}

For the additive group, the same hollow form was used as described above. Furthermore, the cylindrical "silicone bulk" of corresponding dimensions was designed in the CAD software (Fusion 360, Autodesk, San Rafael, USA). The virtual STL file of the modified X-line magnet was submerged in the silicone bulk virtually for the defined depth and subtracted from it using Boolean out function (Fig. 4A). The finished silicone bulk was printed with the ACEO Drop-on-Demand technique with 40A Shore hardness in skin-like color by the manufacturer (ACEO Campus, Burghausen, Germany) (Fig. 4B). The printed silicone bulk was put into the hollow form and the silicone-silicone primer (Multisil-Primer, Bredent, Senden, Germany; LOT 483087) was applied onto the printed silicone and left to dry. The specimen was bonded into the corresponding socket with the same medical-grade conventional silicone (Bredent, Senden, Germany, LOT 450417) as in the conventional group. The whole assembly was put in the oven (Universaloven UN, Memmert, Schwabach, Germany) to polymerize 


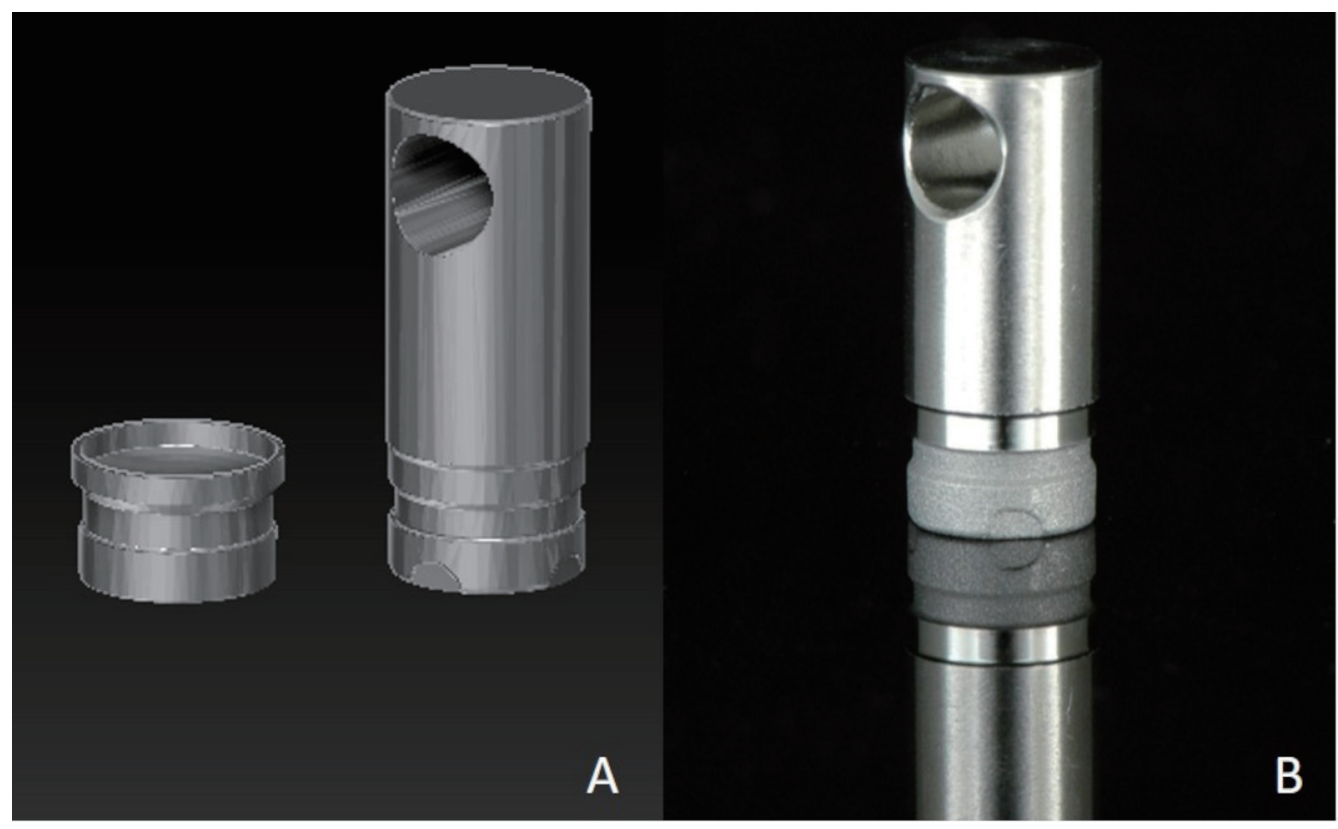

Fig. 2. Design and fabrication of testing specimen. A: virtual design with an extended base $(10 \mathrm{~mm})$ and retention element with transfixion based on the customary X-line magnet (Steco). B: sandblasted X-line magnet with extended base and retention element with transfixion.

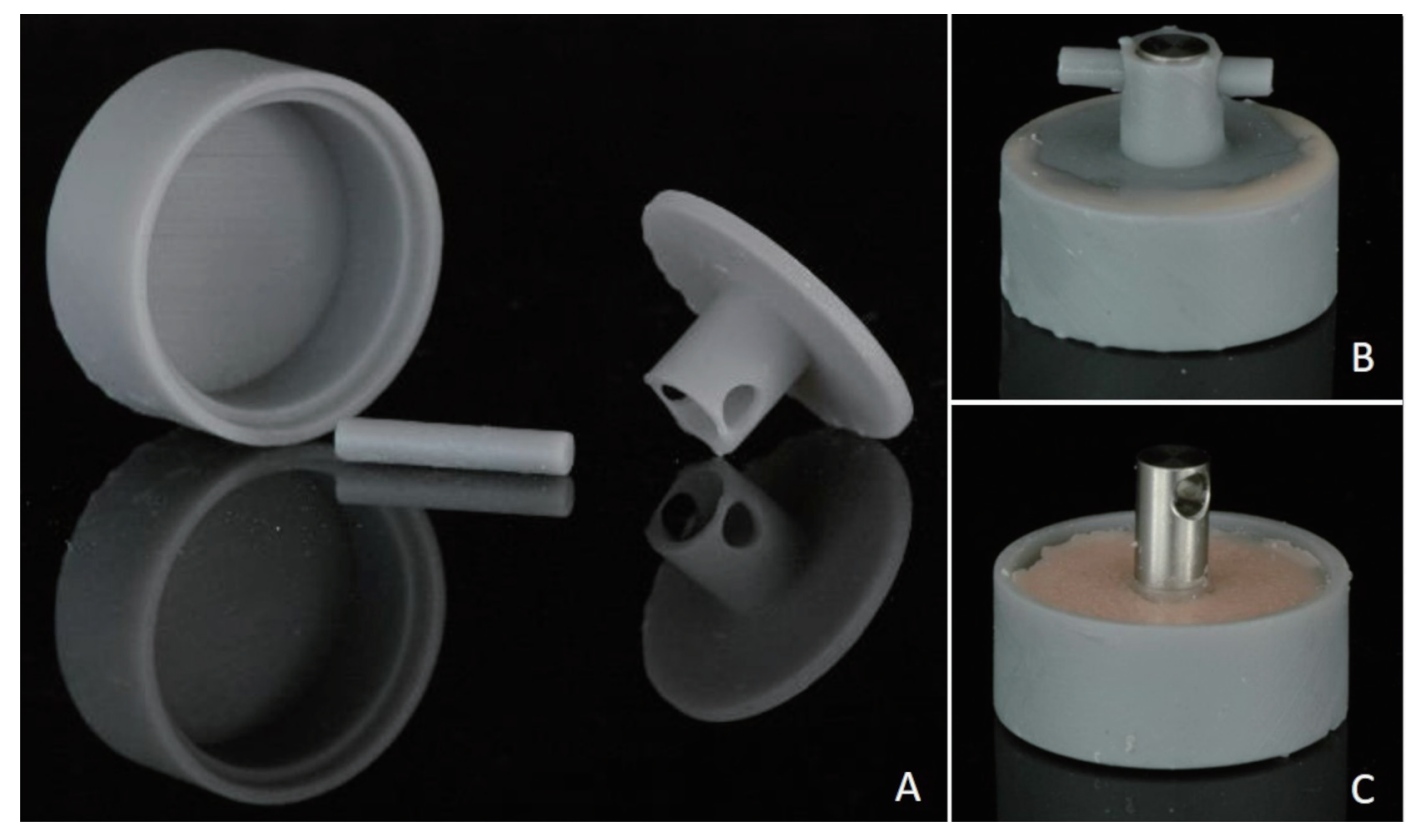

Fig. 3. Embedding the control specimens into silicone. A: retention form with a cover with slot and retention bar for the silicone block manufacturing. B: retention form assembled: the magnet is submerged through the cover into the silicone and fixed by a retention bar. The whole construction is afterwards put in the oven. C: the magnet fixed in the silicone block after polymerization.

the silicone by $60^{\circ} \mathrm{C}$ for 2 hours. 20 such specimens constitute the group "additive-without-primer".

\subsubsection{Application of primer for both groups}

Two metal-silicone primers were applied to twenty test specimens; ten each of: Platinum Primer "G611" (Principality Medical, Newport, UK; LOT B19A) and "A 304"a general purpose primer (Factor II, Lakeside, AZ, USA). The primer fluid was applied on the sandblasted sur- face of each test specimen and left for 10 minutes to dry.

For the conventional group, test specimens were put in conventional silicone blocks, constituting two more groups: conventional-G611 $(n=5)$ and conventional-A304 $(n=5)$. For the additive group, the silicone-silicone primer (Multisil-Primer, Bredent, Senden, Germany; LOT 483087) was applied onto the printed silicone blocks and left dry. Thereafter conventional silicone (Multisil, Bredent, Senden, Germany) was applied into the corresponding magnet socket and the 


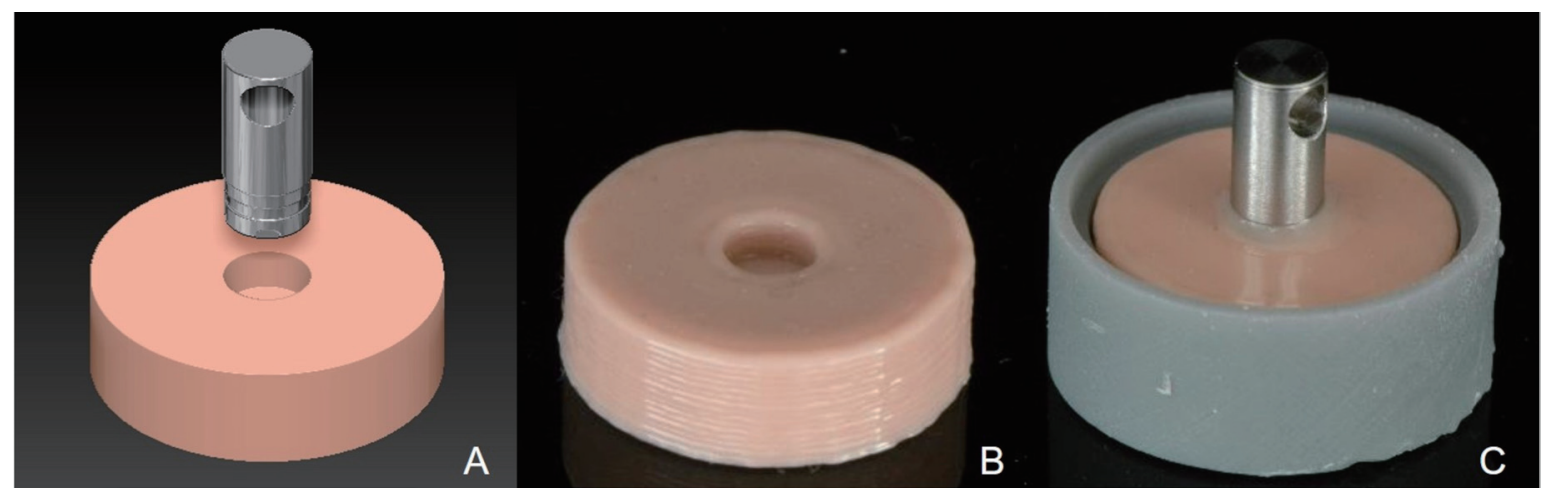

Fig. 4. A: virtual design of the silicone block with a corresponding socket for the X-line magnet. B: 3D printed silicone block. C: the X line magnet applied into the corresponding socket and glued in with conventional silicone

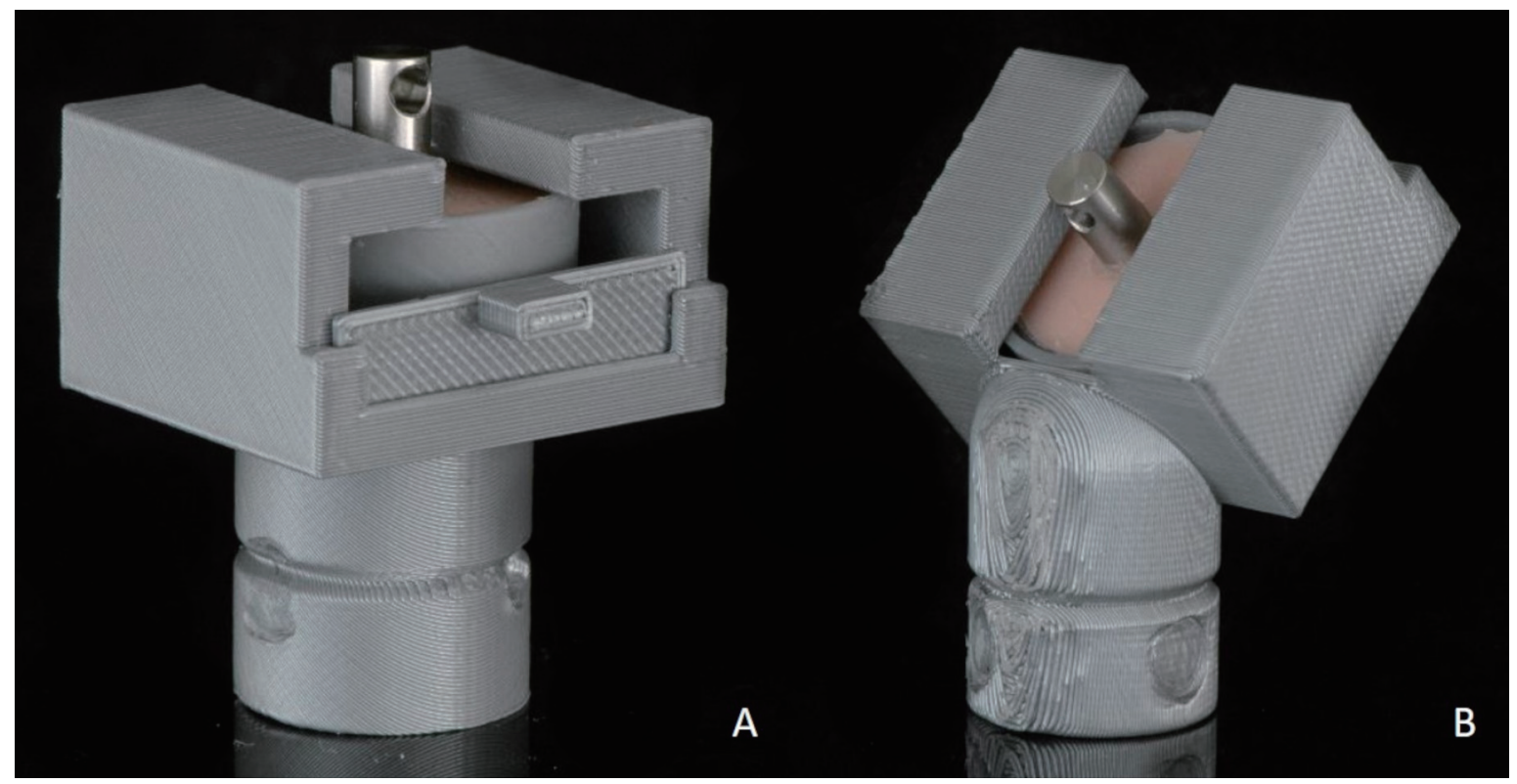

Fig. 5. Retention houses printed with FDM method for $90^{\circ}$ and $45^{\circ}$ angulation tests.

primed specimens were inserted, comprising thereby two more groups: additive-G611 ( $n=5)$, additive-A304 $(n=5)$.

\subsubsection{Retention elements}

Two types of retention houses with $90^{\circ}$ and $45^{\circ}$ angulation towards horizon have been designed for the pull out test. For the additive groups an additional plate has been designed in order to fill the gap between the silicone bulk and its retention house. The retention houses were printed with fused deposition modeling (FDM) printer (Makerbot Replicator 2, Makerbot, NY, USA) and PLA filaments (PLA, Makerbot NY, USA) (Fig. 5).

\subsection{Pull out test}

\subsection{1. $90^{\circ}$ specimens}

The specimens were placed in the retention houses and put in the universal testing machine (Z010, Zwick, Ulm, Germany) (Fig. 6). The screw was inserted into the transfixion of the specimen without ten- sion. The crosshead speed was set on $1 \mathrm{~mm} / \mathrm{min}$. The test was performed until the specimen was fully pulled out from the silicone bulk. The pull out strength (PS) was calculated with the following equation:

$$
P S=\frac{F}{S}
$$

Where $F$ is the maximum pull out force in $\mathrm{N}$ and $\mathrm{S}$ is the square of the attached magnet surface in $\mathrm{mm}^{2}$.

\subsection{Failure type analysis and SEM}

The pulled out magnets (Fig. 7) were qualitatively inspected to define the failure type. Furthermore, scanning electron microscope (SEM) images (LEO 1430; Zeiss; Oberkochen, Germany) were obtained before and after the pull out test to detect any silicone particles on the magnet surface that are not visible at the first sight. 


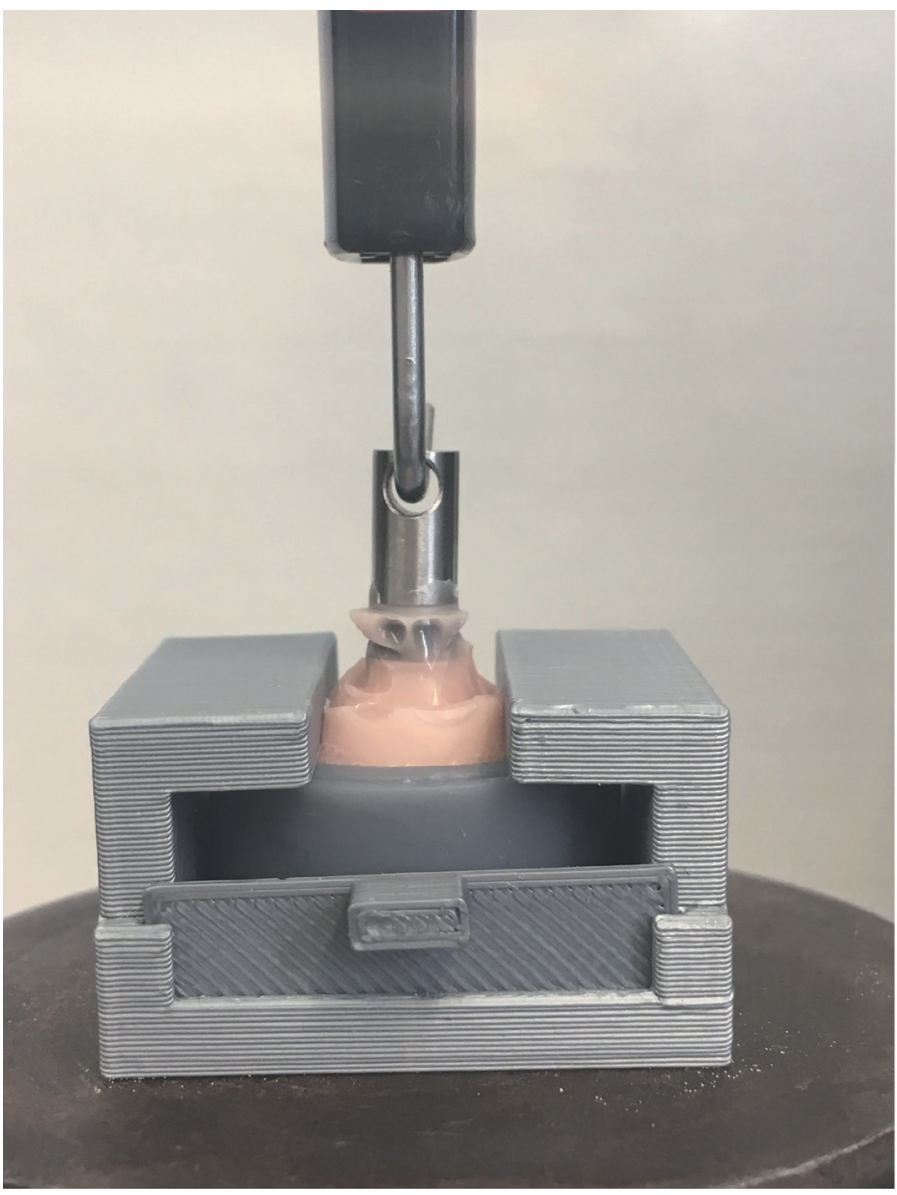

Fig. 6. Pull out test setup in the universal testing machine.

\subsection{Analysis of residual silicone on the specimens}

To measure the amount of residual silicone left on the magnets after the pull out test, the bonding surfaces of the specimens were scanned with a stationary 3D scanner (D2000, 3Shape, Danemark). The obtained STL files were re-segmented in the free form software (Zbrush, Pixologic, Inc., Angeles, CA, USA) to increase the amount of polygons (up to 300 000) in order to enhance the matching accuracy. The resegmented STL files were uploaded in the $3 D$ analyzing software (Geomagic Control X, 3D Systems, Rock Hill, CA, USA) and matched with the initial STL file of the magnet using the "initial fit" and "best fit" functions. The outstanding amount of silicone was calculated as a positive deviation from the initial data and saved in \%, which is a basic function of this software (Fig. 8).

\subsection{Data analysis}

All gathered data was statistically analyzed with JMP 14 (SAS Corp., Heidelberg, Germany). First the data was tested for normality by goodness of fit with Shapiro-Wilk test. For normally distributed groups the statistical difference was tested with Tukey Kramer HSD test; all other with Wilcoxon rank sum test. The significance level for all tests was set on 0.05 .

\section{Results}

\subsection{Pull out test}

The pull out strength values are depicted in Figure 9.

The pull out test without primer revealed significant $(p<0.05)$ difference for $90^{\circ}$ specimens, as the mean pull out strength was $0.17 \pm 0.01$ $\mathrm{N} / \mathrm{mm} 2$ in conventional and $0.11 \pm 0.01 \mathrm{~N} / \mathrm{mm} 2$ in additive group. Significantly $(p<0.05)$ lower values were observed in the $45^{\circ}$ group in the order of $0.03 \pm 0.02 \mathrm{~N} / \mathrm{mm} 2$ in both conventional and additive groups.

The pull out test with primer revealed no significant differences between the G611 and A304 primers in the additive group. However, significantly $(p<0,05)$ higher values were observed for conventional specimens in the A304 group $(1.10 \pm 0.21 \mathrm{~N} / \mathrm{mm} 2)$ compared to the G611 group $(0.59 \pm 0.27 \mathrm{~N} / \mathrm{mm} 2)$.

\subsection{Failure types/SEM}

The SEM pictures of each group of specimens in 40x and 500x magnification are shown in Figure 10. The groups without primer has shown a $100 \%$ adhesive failure type.

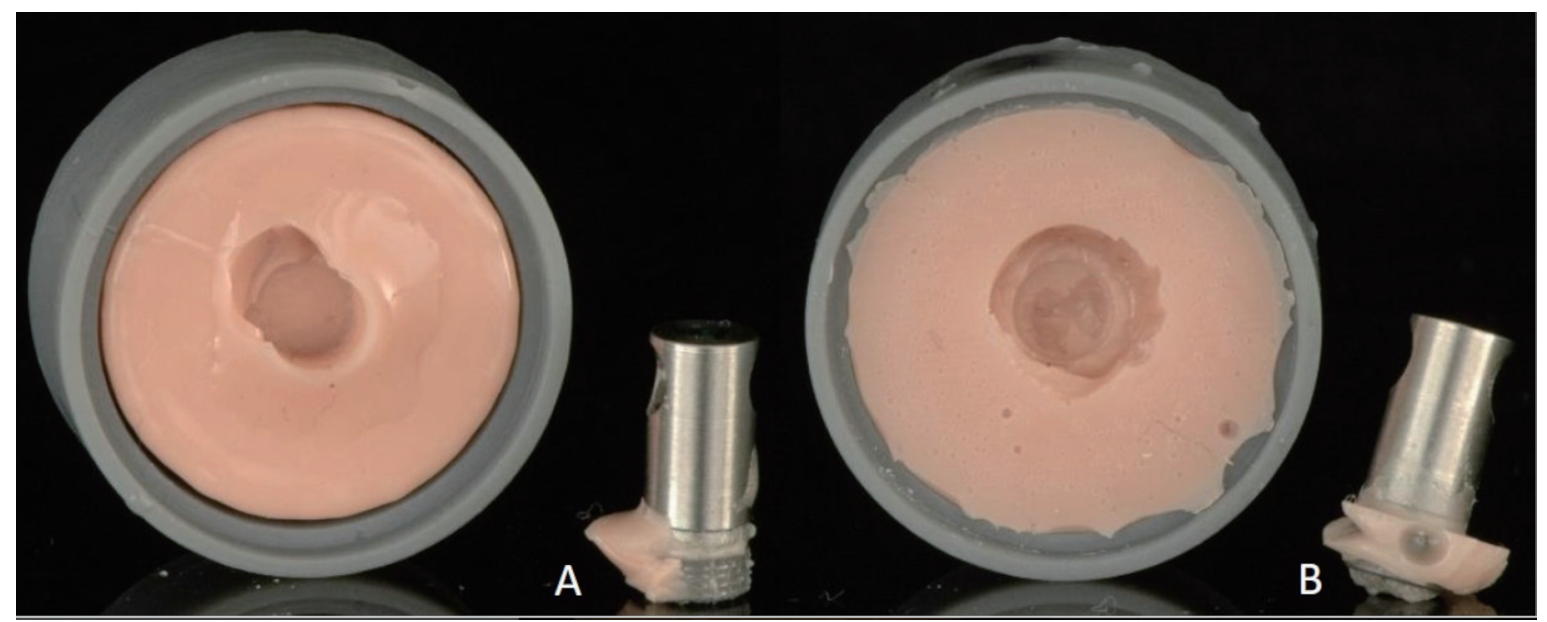

Fig. 7. Example of pulled out X-line magnets from the silicone bulks. A - additive group with primer B - conventional with primer. 


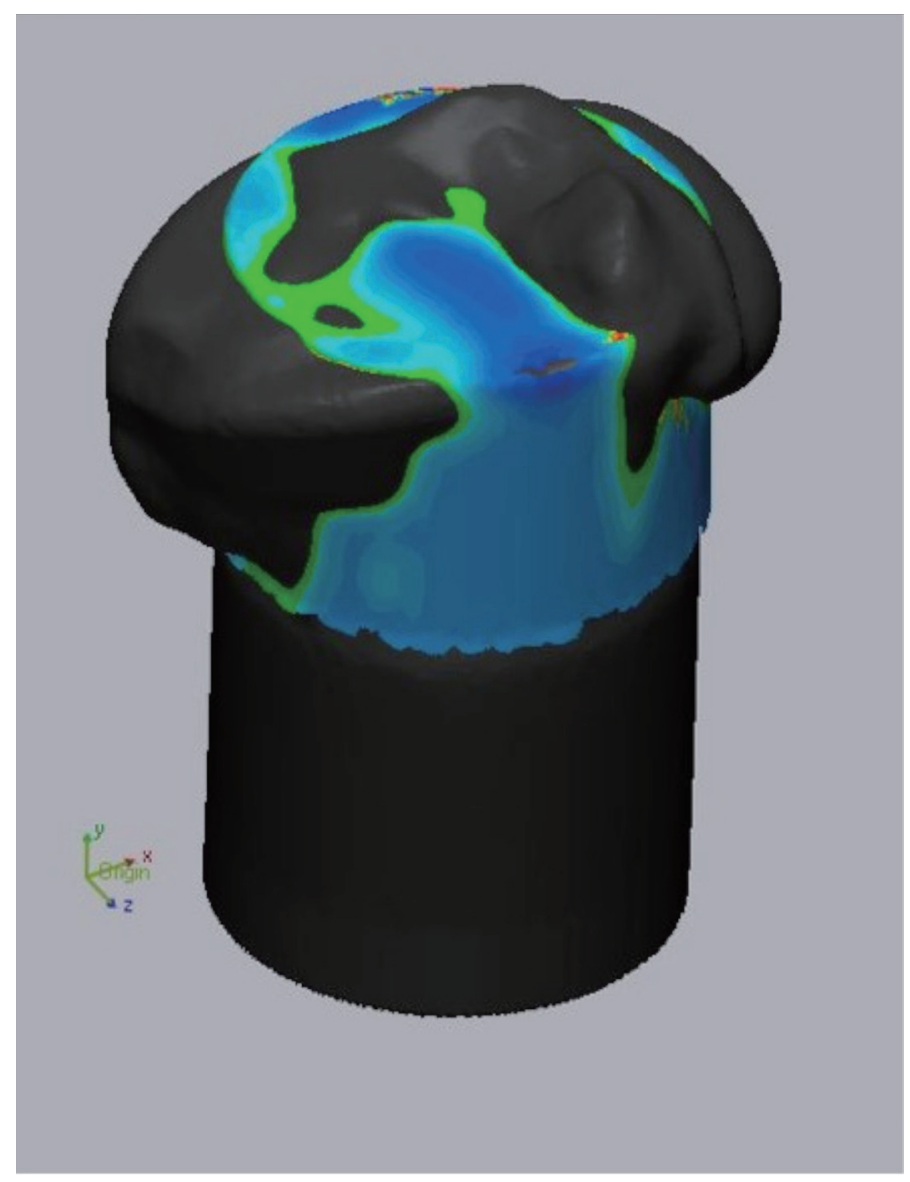

Fig. 8. The scanned specimen with residual silicone matched onto the bonding surface the initial magnet STL file. The blue and green colors indicate no deviation, black indicates silicone bonded to the surface. The square of outstanding amount of residual silicone is calculated in \%.
The examination of specimens with primer under higher magnification has shown that even those specimens, which were considered to have an adhesive failure type, showed in fact the mixed one. The G611 group demonstrated 100\% mixed failure type. The residual silicone particles could be seen evenly distributed on the whole magnet surface (Fig. 10D). However, the conventional specimens demonstrated much bigger parts of silicone residuals, than additive ones (Fig. 10E). In the A304 group also 100\% of specimens demonstrated mixed failure type. On the additive specimens two silicone layers can be observed: fixation layer of conventional silicone and 3D printed silicone attached to each other (Fig. 10H). On the conventional specimens a way bigger residuals can be detected compared to additive group (Fig. 10I).

\subsection{Residual Silicone}

The analysis of residual silicone (Figure 11) revealed no statistical differences between $\mathrm{G} 611$ and A304 primers in conventional group (83.65 \pm 9.02 vs. $76.23 \pm 17.27)$, whereas significantly lower $(p<0.05)$ values were observed for A304 in the additive group (70.15 \pm 5.17 vs. 37.02 \pm 7.66 ).

\section{Discussion}

The results of the study propose the partial rejection of the null hypothesis, as statistically significant higher retention values were observed for conventional silicone with A 304 primer application.

The sufficient retention of magnets within the prosthesis bulk is an important quality requirement to any facial prosthesis, as it significantly increases its longevity and overall clinical performance[12]. A poor chemical retention may facilitate the microleakage of sebum and perspiration into the finish line, causing either further magnet detachment or bacteria accumulation. Both silicone materials applied in this study have been widely used for facial prosthesis manu-

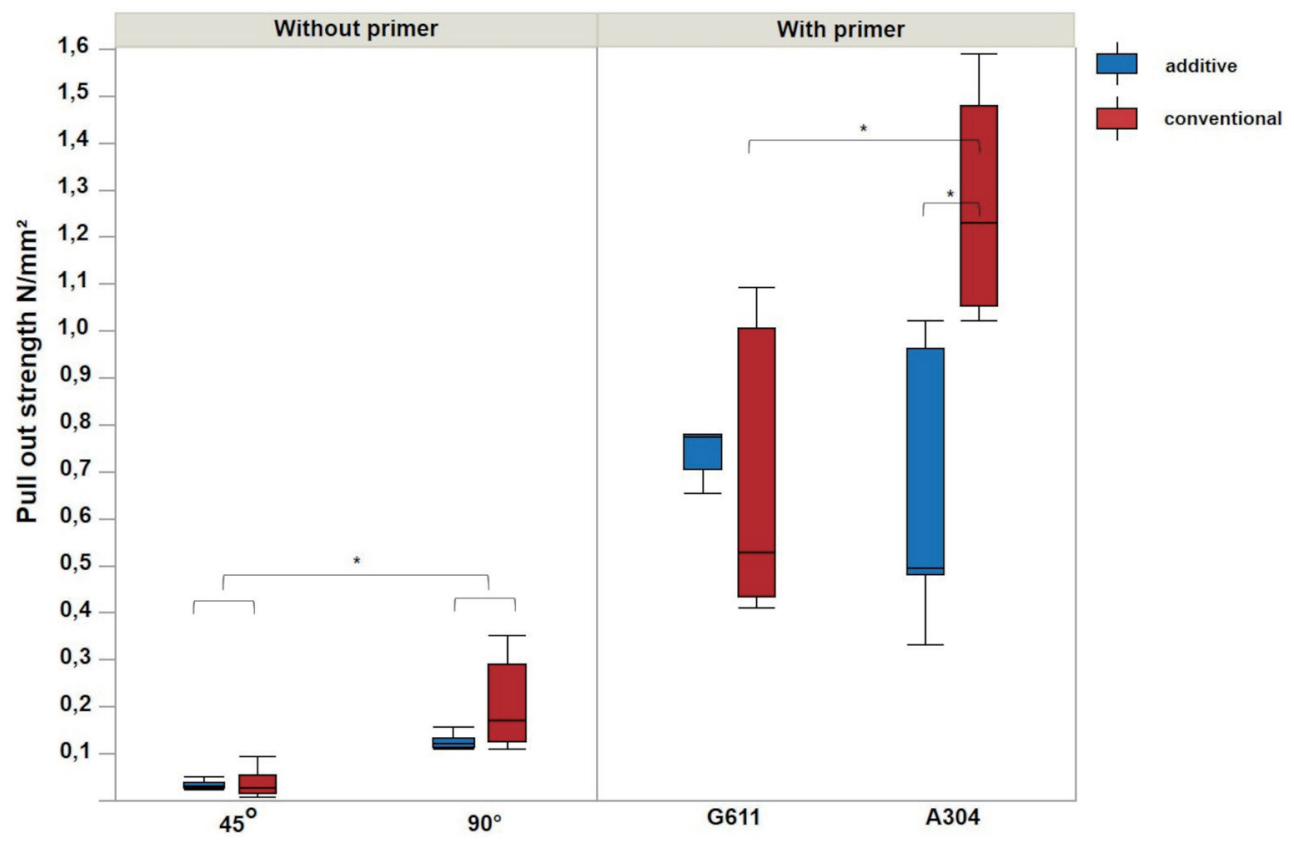

Fig. 9. Pull out strength values of all study groups: $45^{\circ}$ and $90^{\circ}$ without primer and $90^{\circ}$ with $\mathrm{G} 611$ and A304 primers. The * sign indicates the statistically significant difference. 


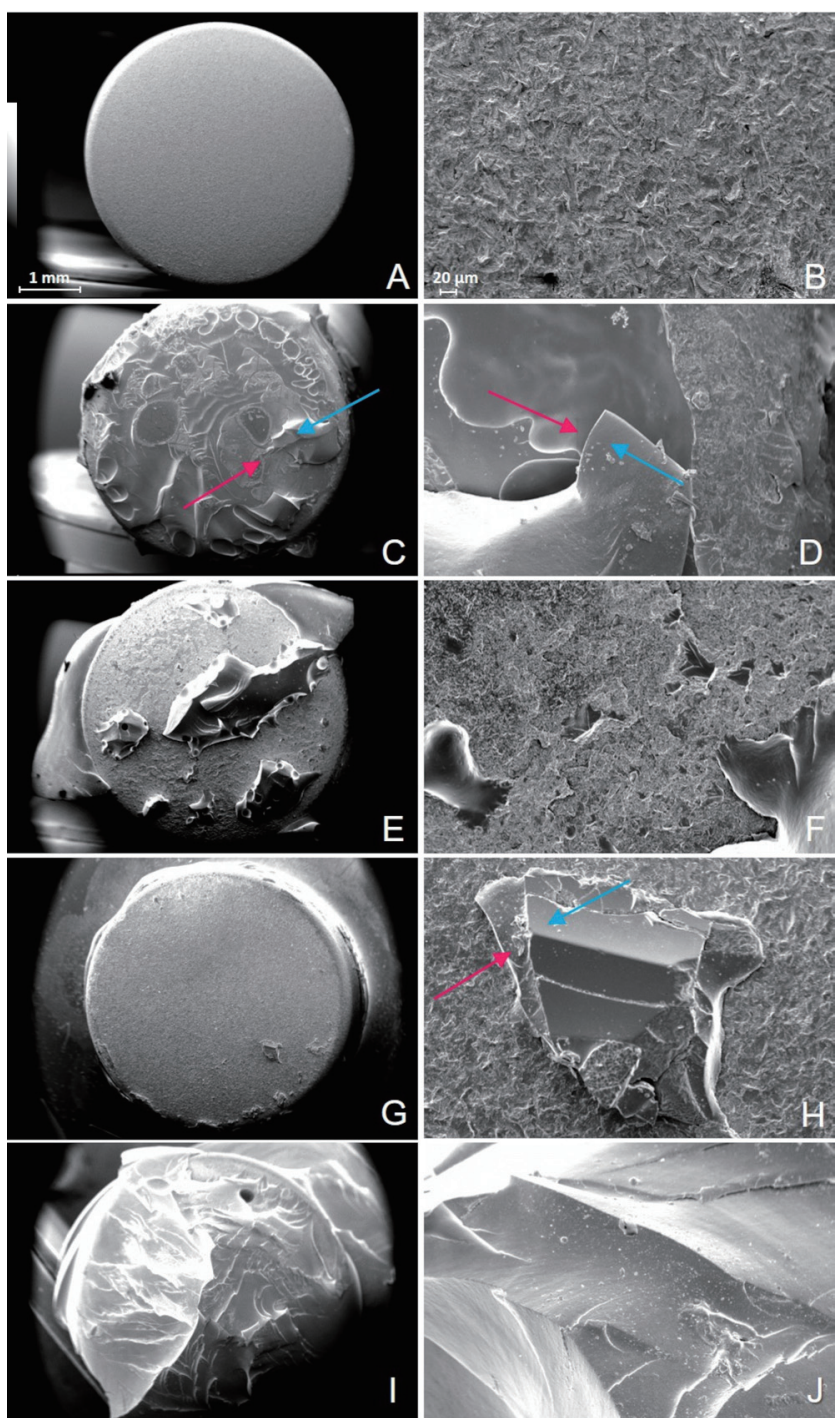

Fig. 10. Figure 10 The SEM images of pulled out magnets with $40 x$ and $500 x$ magnification. The red arrows indicate the residual thin and evenly distributed layer of conventional silicone (used as fixation silicone), and the blue arrows indicate the 3D printed silicone residuals of irregular form.

facturing and the clinical application of 3D printed silicone has been also acknowledged $[6,10]$.

The evaluation of the bond strength between silicone elastomers and titanium magnets may be challenging due to elasticity of silicones and its soft nature. Commonly the $180^{\circ}$ direction is used for the peel test[19-21]. Some other studies utilized the bond strength test for assessing of silicone bonding capacities with $90^{\circ}$ peel design[17]. However, as the force applied to a facial prosthesis while putting it off, may be oriented in various directions, application of the peel force with different level of angulation should be considered in the study design[18]. Thus, Wolf et al. evaluated the peel strength with the $45^{\circ}$ angulation so that the specimens were subjected to the maximal peeling forces. In addition, the tensile test with axially applied force was performed[18]. In the present study the same both test were combined together, as the real retention magnets were submerged in the silicone specimens instead of plain titanium surface. This way the specimens experienced the peeling force with $180^{\circ}$ on the side of the magnet and tensile force on its top with $90^{\circ}$ in case of $90^{\circ}$ applied pulling out force. The pulling out of magnets under $45^{\circ}$ resulted in mixture of shearing, peeling and tensile forces, with a certain degree of rotation. Such testing may resemble the forces applied to prosthesis, when putting it off in a clinical situation. This fact may explain the way lower bond strength in the $45^{\circ}$ test and proposes the utilization of such angulation for further studies to the topic.

As the present study exploited an alternative test design, than similar studies to the topic, a comparison of absolute bond strength values requires a careful interpretation. Thus, in 2001 the Wolf et al. reported the highest tensile bond strength (TBS) to titanium of $0.2 \mathrm{~N} / \mathrm{mm}^{2}$ for silicone material, which is way lower as in the present study[18]. Artopolou et al. has shown the peel bond strength (PBS) values of $1.05 \mathrm{~N} / \mathrm{mm}^{2}$ for polyvinylsiloxane elastomers using the same A304 primer[17]. However, due to the Weibull analysis the value of $63.2 \%$ must be considered, meaning the real PBS values might be in fact greater, which restricts an objective comparison to the present study.

The clinical interpretation of the reported bond strength values is also of importance. On the one hand, in current literature no guidelines can be found to determine the threshold of clinical relevance and, which bond strength values should be considered as sufficient for the clinical use. On the other hand, the bond strength values between silicone elastomers and titanium can be compared to those of acrylic superstructures. Thus, Hatamleh et al. reported the PBS values from $0.85 \pm 0.12$ to $1.78 \pm 0.47 \mathrm{~N} / \mathrm{mm}^{2}$ for three maxillofacial silicone elastomers bonded to acrylic substrate in combination with A 304 primer[14]. These values are quite comparable to $1.1 \pm 0.21$ $\mathrm{N} / \mathrm{mm}^{2}$ as revealed in the present study, which may indicate bond strength between titanium and silicone elastomers used in the present case is sufficient for the clinical application. In the additive group the PBS values in axial direction of force, ranged between 0.64 and $0.65 \mathrm{~N} / \mathrm{mm}^{2}$, which was - basing on the values reported in the above mentioned studies - assumed to be clinically acceptable. However, only in vivo studies may prove this assumption. In the conventional group the A304 demonstrated higher PBS values than G611, which were even greater, as in most of the reported studies on this topic, especially with regard to non-axial occurring forces.

The two silicone materials used in the study have the same polyvinylsiloxane composition. For this reason, the differences in the bond strength within each test group are attributed rather to the primer composition and its chemical affinity to the silicone substrates. This can be attributed to the composition of the A304 platinum primer, consisting of naptha (85\%), tetra-n-propyl silicate (5\%), tetrabutyltitanate (5\%) and tetra (2-methoxyethoxy) silane (5\%) and the G611 includes propan-2-ol and various vinyl silanes.

The shore $A$ hardness has been shown to have a positive correlation with the bond strength to maxillofacial silicone elastomers[19]. In previously reported cases the silicone auricular prostheses was printed in 60A hardness to facilitate the better stability[11]. However, due to the fact that conventional silicone has the 40A shore hardness the additively produced silicone was also printed in 40A, in order to standardize the study measures. The study of Hatamleh et al. has shown that the bonding between resin substrate and silicone is sufficient enough for the clinical use and a greater retention can be achieved rather by improving the silicone mechanical properties[19].

The analysis of the SEM images of specimens with primer application exposed a "small island" of residual silicone over the whole magnet 


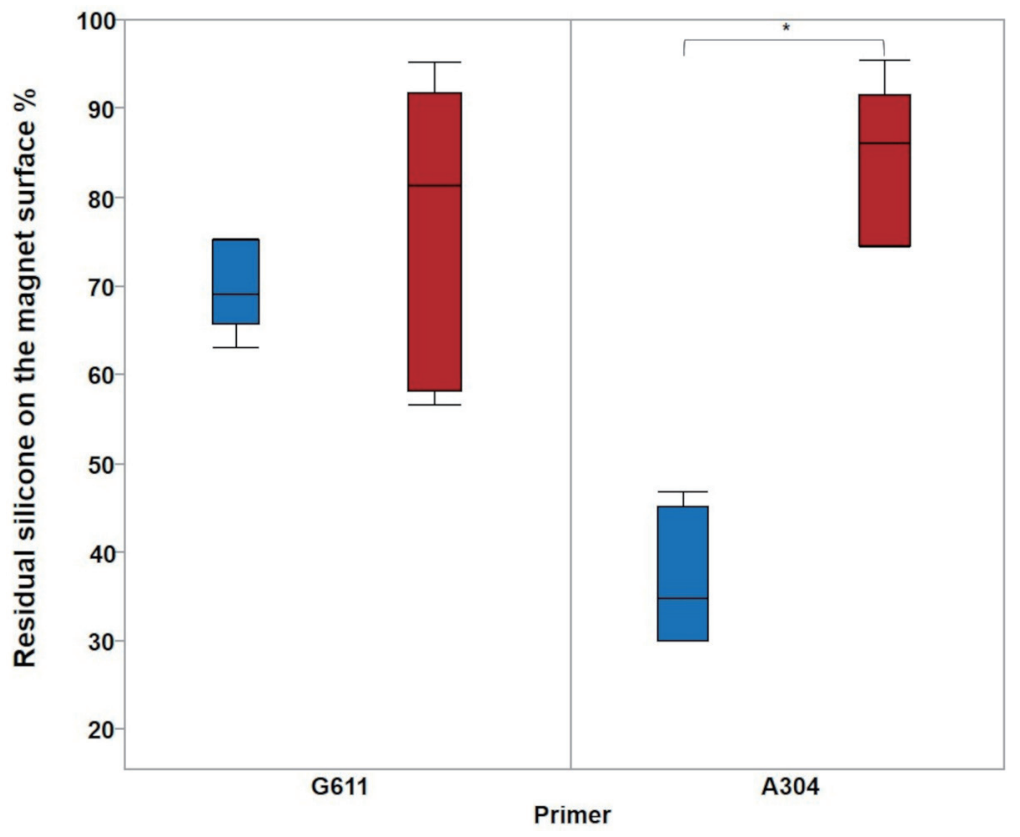

Fig. 11. The amount of residual silicone on the magnet surface after the pull out test in \% for G611 and A304 primers.

surface in all primer study groups. This means, that in fact there were more silicone residuals than the 3D analysis has detected. However, the overall tendency may remain the same between all study groups. Nearly the same amount (72\%) of residual silicone after A304 Primer application was reported in the study of Artopoulou et al[17]. However, in case of A304 primer, more silicone residuals have been observed on the magnet surface after the pull-out test in conventional group. This may be also attributed to the way the magnet was fixed within the silicone specimens' bulk. Thus in the additive group only a thin layer of conventional silicone was applied into the printed silicone bulk to fix the magnet. Furthermore, at the CAD stage no additional space was designed for this fixing conventional silicone layer, which also contributed to its very thin distribution within the printed silicone bulk. In contrast, in conventional group the magnet was surrounded by a thicker layer of conventional silicone.

Basically, mixed failure types indicate that the bond strength between silicone and titanium is in some cases stronger, than the tear strength of the silicone. In the additive group both silicone layers (conventional silicone with adhesively bonded parts of the 3D printed bulk) were attached to the surface of cpTi's. This indicates that the bond strength between the printed and conventional silicone is sufficient enough to allow a clinically acceptable integration of retention magnets into the printed silicone. Application of other adhesives, such as A-100 (Medical adhesive type A, Technovent, Bridgent, South Wales, UK) might be tested in future studies.

Extraoral, human, and environmental conditions has been shown to influence the mechanical properties of maxillofacial silicone elastomers[22]. In the present study no artificial aging was performed, which should be considered as its limitation. The influence of hydrolytic or environmental degradation due to air, sun, sweat and perspiration exposure on the silicone retention capabilities should be considered in further studies.
The present study assessed the new retention concept for the additively manufactured facial prostheses in a fully digital workflow. An adhesive anchorage of retention magnets directly within a printed silicone bulk constitutes a quicker and technically simplified approach, in comparison to utilization of an acrylic retention superstructure. This way, due to the silicone elasticity, the retention magnets have the certain degree of freedom within the prosthesis bulk, which facilitates the passive fit of a facial prosthesis. The passivation is certainly not the major concern in the extraoral prosthetics, as no functional load of a facial prosthesis is expected. Still, this aspect may be considered as beneficial towards the acrylic retention bar. Further clinical studies are necessary to assess the long-term performance of such retention concept.

In the present study the cpTi magnets were not subjected to any additional physico-chemical treatment and have been utilized in a sandblasted state, as they are delivered by the manufacturer. Thus, it remains unclear, if a change of design towards surface area enlargement to rise physical retention (e.g. grooves) might be beneficial. Even though, whether an application of tribochemical coating or plasma surface treatment may aid a better retention between cpTi and silicone should be investigated in further studies to the topic. Furthermore, due to different regulations of medical device design/ allowance, the utilization of stainless steel coated magnets for the investigated concept of magnet retentions should be also taken into account in further research.

\section{Conclusion}

The findings of the present study propose that in general any retention system experiences higher stress in the $45^{\circ}$ angulation, than during axially applied pulling forces. Both primers utilized in the present study yielded a potentially clinically acceptable retention under the $90^{\circ}$ testing conditions, based on the values reported in previous studies. The A304 primer may allow a silicone bonding higher than 
G611 primer. The adhesive anchorage of retention magnets in a directly printed silicone prosthesis using the polyvinylsiloxane-based elastomer may be considered as a valid technical approach and applied in a digital workflow of facial prostheses manufacturing. Further in vivo studies are needed to prove this technical approach and threshold clinically, especially considering angulated forces.

\section{Acknowledgements}

The authors thank Mrs. C. Schille, Mr. E. Schweitzer and Prof. Dr. J. Geis-Gerstdorfer for their technical support and scientific contribution to the study. The special thanks goes to Mr. S. Metzner from Steco $\mathrm{GmbH}$ and Mr. P. Brehm from Bredent $\mathrm{GmbH}$ for their technical support and materials supply.

\section{Conflicts of interest}

The authors report no conflict of interest.

\section{References}

[1] Tetteh S, Bibb RJ, Martin SJ. Maxillofacial prostheses challenges in resource constrained regions. Disabil Rehabil. 2019;41:348-56. https://doi.org/10.108 0/09638288.2017.1390697, PMID:29065718

[2] Phasuk K, Haug SP. Maxillofacial Prosthetics. Oral Maxillofac Surg Clin North Am. 2018;30:487-97. https://doi.org/10.1016/j.coms.2018.06.009, PMID:30266191

[3] Elbashti M, Sumita Y, Kelimu S, Aswehlee A, Awuti S, Hattori M, et al. Application of Digital Technologies in Maxillofacial Prosthetics Literature: A 10-Year Observation of Five Selected Prosthodontics Journals. Int J Prosthodont. 2018;32:45-50. https://doi.org/10.11607/ijp.5932, PMID:30372513

[4] Unkovskiy A, Roehler A, Huettig F, Geis-Gerstorfer J, Brom J, Keutel C, et al. Simplifying the digital workflow of facial prostheses manufacturing using a three-dimensional (3D) database: setup, development, and aspects of virtual data validation for reproduction. J Prosthodont Res. 2019;63:313-20. https://doi.org/10.1016/j.jpor.2019.01.004, PMID:30792148

[5] Elbashti M, Itamiya T, Aswehlee A, Sumita Y, Ella B, Naveau A. Augmented Reality for Interactive Visualization of 3D Maxillofacial Prosthetic Data. Int J Prosthodont. 2020;33:680-3. https://doi.org/10.11607/ijp.6835, PMID:NOT_ FOUND

[6] Unkovskiy A, Brom J, Huettig F, Keutel C. Auricular Prostheses Produced by Means of Conventional and Digital Workflows: A Clinical Report on Esthetic Outcomes. Int J Prosthodont. 2018;31:63-6. https://doi.org/10.11607/ ijp.5446, PMID:29145528

[7] McHutchion L, Aalto D. Simulation of tissue-prosthesis margin interface by using surface scanning and digital design for auricular prostheses. J Prosthet Dent. 2021;125:361-72. https://doi.org/10.1016/j.prosdent.2020.01.045 PMID:32336538
[8] Nuseir A, Hatamleh MM, Alnazzawi A, Al-Rabab'ah M, Kamel B, Jaradat E. Direct 3D Printing of Flexible Nasal Prosthesis: Optimized Digital Workflow from Scan to Fit. J Prosthodont. 2019;28:10-4. https://doi.org/10.1111/ jopr.13001, PMID:30461125

[9] Unsal GS, Turkyilmaz I. Improved reconstruction of an implant-retained auricular prosthesis using CAD/CAM technology. J Dent Sci. 2019;14:328-9. https://doi.org/10.1016/j.jds.2019.02.002, PMID:31528263

[10] Unkovskiy A, Spintzyk S, Brom J, Huettig F, Keutel C. Direct 3D printing of silicone facial prostheses: A preliminary experience in digital workflow. J Prosthet Dent. 2018;120:303-8. https://doi.org/10.1016/j.prosdent.2017.11.007, PMID:29429837

[11] Unkovskiy A, Wahl E, Huettig F, Keutel C, Spintzyk S. Multimaterial 3D printing of a definitive silicone auricular prosthesis: an improved technique. J Prosthet Dent. 2021;125:946-50. https://doi.org/10.1016/j.prosdent.2020.02.021 PMID:32680736

[12] Daniel S. Towards Developing CAD/CAM Solutions in the Retention of Extra-Oral Facial Prosthetics PhD Thesis. 2014.

[13] Kincade C, McHutchion L, Wolfaardt J. Digital design of patient-specific abutments for the retention of implant-retained facial prostheses. J Prosthet Dent. 2018;120:309-12. https://doi.org/10.1016/j.prosdent.2017.10.002, PMID:29526301

[14] Hatamleh MM, Watts DC. Bonding of maxillofacial silicone elastomers to an acrylic substrate. Dent Mater. 2010;26:387-95. https://doi.org/10.1016/j. dental.2010.01.001, PMID:20122716

[15] Polyzois G, Pantopoulos A, Papadopoulos T, Hatamleh M. Effect of light aging on silicone-resin bond strength in maxillofacial prostheses. J Prosthodont. 2015;24:215-9. https://doi.org/10.1111/jopr.12202, PMID:25081922

[16] Kantola R, Lassila L, Vallittu P. Adhesion of maxillofacial silicone elastomer to a fiber-reinforced composite resin framework. Int J Prosthodont. 2011;24:582-8. PMID:22146259

[17] Artopoulou II, Chambers MS, Zinelis S, Eliades G. Peel strength and interfacial characterization of maxillofacial silicone elastomers bonded to titanium. Dent Mater. 2016;32:e137-47. https://doi.org/10.1016/j.dental.2016.03.024, PMID:27118682

[18] Wolf BH, Reitemeier BK, Schmidt AE, Richter GH, Duncan G. In vitro testing of the bond between soft materials used for maxillofacial prostheses and cast titanium. J Prosthet Dent. 2001;85:401-8. https://doi.org/10.1067/ mpr.2001.114511, PMID:11319539

[19] Hatamleh MM, Watts DC. Mechanical properties and bonding of maxillofacial silicone elastomers. Dent Mater. 2010;26:185-91. https://doi. org/10.1016/j.dental.2009.10.001, PMID:19892390

[20] Güngör MBANKOĞLU, Nemli SKARAKOCA, İnal CB, Bağkur M, Dilsiz N. Effect of plasma treatment on the peel bond strength between maxillofacial silicones and resins. Dent Mater J. 2020;39:242-50. https://doi.org/10.4012/ dmj.2018-259, PMID:31776314

[21] Haddad MF, Goiato MC, Santos DM, Crepaldi NM, Pesqueira AA, Bannwart LC. Bond strength between acrylic resin and maxillofacial silicone.J ApplOral Sci. 2012;20:649-54. https://doi.org/10.1590/S1678-77572012000600010, PMID:23329247

[22] Hatamleh MM, Polyzois GL, Nuseir A, Hatamleh K, Alnazzawi A. Mechanical Properties and Simulated Aging of Silicone Maxillofacial Elastomers: Advancements in the Past 45 Years. J Prosthodont. 2016;25:418-26. https:// doi.org/10.1111/jopr.12409, PMID:26805510

This is an open-access article distributed under the terms of Creative Commons Attribution-NonCommercial License 4.0 (CC BYNC 4.0), which allows users to distribute and copy the material in any format as long as credit is given to the Japan Prosthodontic Society. It should be noted however, that the material cannot be used for commercial purposes. 